\title{
44. The Effects of Hypothermia of Cession of Postencephalitic Epilepsy
}

\author{
Terukazu ImaI, Terumi Uchiyama and Masao RaI \\ Division of Surgery, Tukaguchi Branch of Amagasaki Prefectural Hospital
}

The patient suffered from encephalitis japonica 4 yars ago. The epileptic convulsion began about 3 years ago and increased gradually in frequency. After convulsion, the patient always complained of hadache, diplopia and numbness of the left side of the body. Various kinds of anticonvalsant drugs were given to the patient during 3 years, but there were no effects on the prevention of an attack of epilepsy. About 6 months ago the patient affected with a status epilepticus continuing for 4 days.

The physical and neurologic findings during the intervals of convulsions were normal but the right oculomotorius paresis. The bilateral ventricles were slightly but diffusely enlarged after carotid angiography and lumbar pneumoencephalography. Dysrhythmia was noted bilaterally after electroencephalography. The patient was anesthesized with GOF by intubation for 2 hours to disrupt convulsion. After waking up from GOF anesthesia, the patient affected with epilepsy again. The body cooling was then, carried out for 3.5 hours in the patient under intubations anesthesia with GOF. The temperature of the rectum was $29^{\circ}-30^{\circ} \mathrm{C}$. No epileptic convulsion occurred for these 6 months after once of the body cooling. At present, no neurological sequelae are found. The anticonvalsant drugs have been continuously given to the patient.

From the results of this case, it is thought that hypothermia would effect on the prevention of the epileptic attack.

\section{Subcortical or Brainstem Lesion Effect on Subcorticogenic Seizure with Special Reference to the Forel-H Field}

-experimental analysis-

\author{
Dennosuke Jinnai, Heitaro Mogami, Jiro Mukawa, Takuya Ikeda \\ and Yoshikazu Iwata \\ Department of Neurosurgery, Osaka University Medical School
}

1. Chronic experiment: Utilyzing unanesthetize cats, an attempt was done to make an electroencephalographical analysis of the chronic effect of the bilateral 